\title{
MODELOS ECOMORFOLÓGICOS PARA VERTEBRADOS ARBORÍCOLAS: O CASO DO MARSUPIAL PHILANDER FRENATA.
}

\section{ECOMORPHOLOGICAL MODELS FOR ARBOREAL VERTEBRATES: THE CASE OF THE MARSUPIAL PHILANDER FRENATA.}

\section{Delciellos, A. C. ${ }^{1}$ and Vieira, M. V. ${ }^{2}$}

1 acidadela@bol.com.br $;{ }^{2}$ mvvieira@biologia.ufrj.br

1, 2 Laboratório de Vertebrados, Departamento de Ecologia, UFRJ, CP 68020, Rio de Janeiro - RJ, 21941-590

\section{RESUMO}

Medidas de desempenho utilizadas podem fornecer uma medida do nicho fundamental, permitindo prever uso do habitat ou recursos na ausência de interações entre espécies. Propomos um conjunto padrão de testes de desempenho locomotor para vertebrados arborícolas, e um método para desenvolver modelos ecomorfológicos baseados em testes de desempenho e análise de caminhos. Os testes propostos simulam características do habitat como diâmetro e orientação de suportes. Modelos de relações entre variáveis podem ser formulados e comparados através da análise de caminhos, permitindo escolher o modelo de melhor ajuste aos dados. Este procedimento foi aplicado ao marsupial Philander frenata, comparando-se o efeito da forma corporal sobre o comprimento e freqüência de passadas no andar arborícola. $\mathrm{O}$ modelo incluindo caminhos tanto para freqüência como para comprimento de passadas teve um ajuste significativamente melhor que o modelo com caminhos apenas para o comprimento das passadas. Um modelo gerado a posteriori, a partir da eliminação de caminhos nãosignificativos, sugere que o comprimento relativo das garras e da cauda são as variáveis que mais influenciam a freqüência de passadas, enquanto o comprimento das passadas é mais afetado pelo comprimento relativo dos membros. Estas são hipóteses sobre um aspecto importante do nicho potencial de marsupiais didelfídeos, que podem permitir 
inferir similaridade de nicho fundamental a partir da morfologia. Dados independentes são necessários para testar estas hipóteses.

Palavras-chave: Análise de caminhos, marsupiais, locomoção, ecomorfologia, Philander frenata

\section{ABSTRACT}

Performance measurements can be used to infer the fundamental niche of species, allowing predictions of habitat or resource use in the absence of species interactions. We propose a standard set of tests to measure locomotory performance of arboreal vertebrates, and a procedure to develop ecomorphological models based on performance tests and path analysis. The proposed tests simulate habitat features such as support diameter and incline. Models of relationships between variables can be formulated, compared with path analysis, and the model of best fit chosen. This procedure was applied to the didelphid marsupial Philander frenata, comparing the effect of body shape on stride length and frequency in arboreal walking. The model including paths to stride length and frequency had a significant better fit than the model with paths only to stride length. An a posteriori model - obtained from the elimination of nonsignificant paths - suggested that the relative length of claws and tail were the more determinant of stride frequency, whereas stride length was more affected by the relative length of the limbs. These are hypotheses about an important aspect of the fundamental niche of didelphid marsupials, allowing inferences of niche similarity based on morphology. Independent data are necessary to test these hypotheses.

Keywords: Path analysis, marsupials, locomotion, ecomorphology, Philander frenata

\section{INTRODUÇÃO}

Nas décadas de 70 e 80 uma série de estudos sobre partilha de recursos gerou uma grande quantidade de dados de campo sobre nicho e estrutura de comunidade (SCHOENER, 1989). Estes estudos e aplicações se restringiram ao nicho realizado ("realized niche"), isto é, o nicho realizado como produto final da interação do organismo com o ambiente, seus predadores, parasitas e competidores (HUTCHINSON, 1957). Por dificuldades óbvias, raros foram os estudos que mediram o nicho potencial ou fundamental, o nicho de um organismo na ausência de interações com outras espécies em uma situação natural (HUTCHINSON, 1957). Apenas em laboratório, na ausência destas interações, seria possível uma medida de nicho potencial. Com ela seria possível construir um modelo nulo para a estrutura de comunidade, que permitiria prever a estrutura da comunidade baseada somente em limitações das espécies no uso de hábitat e 
recursos, como se não houvesse interações entre espécies (RICKLEFS \& MILES, 1994). Um dos caminhos recentes para chegar a este tipo de modelo nulo é a ecomorfologia (MOERMOND, 1986; POUNDS, 1991; RICKLEFS \& MILES, 1994; SCHOENER, 1989).

Uma abordagem ecomorfológica recente soma medidas de desempenho ("performance") às medidas morfológicas e ecológicas (WAINWRIGHT \& REILLY, 1994). O desempenho é uma medida da capacidade máxima do organismo em uma atividade específica, como por exemplo, a velocidade máxima no solo ou a quantidade de alimento que o organismo encontra por unidade de tempo (GARLAND \& LOSOS, 1994; HERNANDEZ \& MOTTA, 1997; LOSOS, 1990).

A morfologia afeta a ecologia de um organismo indiretamente, através do desempenho que permite em tarefas relacionadas ao uso do habitat, isto é, no uso de recursos. O uso de recursos, por sua vez, determina a sobrevivência e reprodução, que por fim afetam a aptidão, ecologia das populações e comunidades (Figura 1). Assim, medidas de desempenho podem ser vistas como medidas do nicho potencial, que não podem ser avaliadas medindo-se em campo, por exemplo, o uso do habitat e dieta. Através da comparação de medidas de desempenho seria possível estimar diferenças no nicho potencial entre espécies (RICKLEFS \& MILES, 1994).

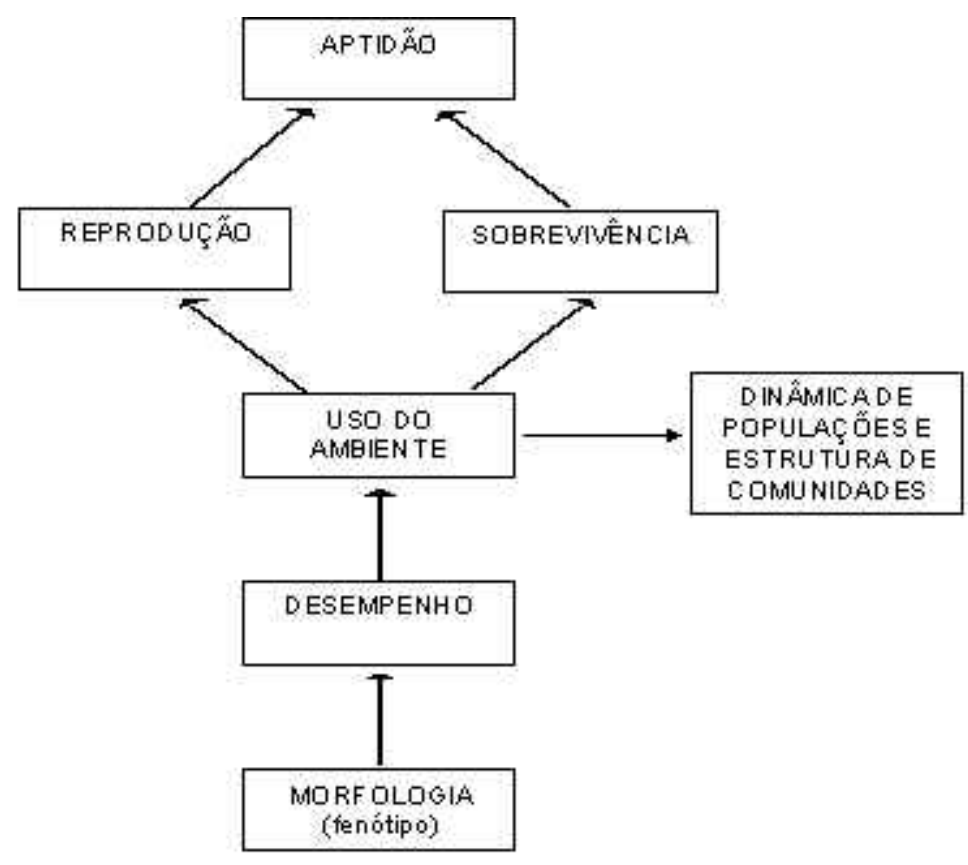

Figura 1: Caminhos que efeitos da morfologia seguem até afetar aptidão, ecologia de populações e comunidades. Modificado de Ricklefs \& Miles (1994).

Estudos sobre a relação entre ecologia e morfologia de primatas (CANT, 1992) e lagartos arborícolas (POUNDS, 1988) demonstraram que o tamanho corporal e o comportamento postural são os principais fatores que vão influenciar a capacidade de aquisição de alimento e a fuga de predadores. Algumas das propriedades do dossel como 
o diâmetro, a densidade de suportes, e seu grau de abertura, podem tornar-se problemáticas conforme o aumento do tamanho corporal. A estrutura do habitat pode apresentar obstáculos para movimentos efetivos e aquisição de comida (CANT, 1992; VIEIRA, no prelo).

Assim, as diferenças encontradas entre espécies podem ser explicadas como resultado de várias pressões seletivas que esses animais sofrem devido à vida arborícola, como (1) andar seguramente, reduzindo as chances de cair; (2) desenvolver velocidade para captura de alimento ou fuga de predadores; (3) escalar suportes largos, para alcançar alimento, ou simplesmente locomover-se; (4) escalar suportes finos, que envolvem soluções diferentes dos largos; (5) cruzar descontinuidades na copa, como distâncias entre galhos diferentes ou árvores diferentes; (6) encurtar o caminho ao longo dos galhos de uma árvore, de forma a cobrir distâncias rapidamente; (7) alcançar frutos ou alimento localizado em ramos terminais, finos; e (8) capturar presas móveis.

Os itens 3 a 6 estariam diretamente relacionados ao encurtamento do caminho percorrido pelo animal, e adicionando-se o aumento da velocidade (2) resultaria na minimização dos gastos de energia e tempo, e na maximização da obtenção do alimento, que pode ser vantajosa dependendo da estratégia de forrageamento do animal. Os problemas relacionados acima acabam gerando e refinando soluções morfológicas, fisiológicas, e comportamentais para problemas que esses organismos confrontam em busca do sucesso reprodutivo (CANT, 1992).

O objetivo deste trabalho é testar uma metodologia para o desenvolvimento de modelos ecomorfológicos relacionando variáveis morfológicas, de desempenho e de uso do ambiente, especialmente para animais arborícolas. O desempenho relativo de diferentes espécies ou indivíduos nestas medidas pode ser usado então como uma medida de nicho potencial.

\section{TESTES DE DESEMPENHO LOCOMOTOR PARA ANIMAIS ARBORÍCOLAS}

Estudos experimentais das relações funcionais entre as características estruturais do habitat e locomoção podem ser realizados em laboratório, usando estudos de observação no campo para guiar o design de testes de desempenho locomotor. Estes testes podem ser realizados com um aparato experimental simples, simulando troncos, galhos e cipós, mas de diâmetro e textura de superfície passíveis de padronização, como tubos de PVC, cordas, madeirame e ferragens disponíveis comercialmente. A textura da superfície dos suportes ("galhos" ou "troncos" de PVC) pode ser padronizada recobrindo os suportes com com fita crepe, que aumenta a aderência dos animais e padroniza a aderência ao substrato. Outros materiais são possíveis mas deve-se lembrar sempre que são testes de desempenho, isto é, o animal deve ser levado ao extremo da sua capacidade. 
A locomoção ao longo dos suportes é gravada com uma câmera de vídeo, para medir posteriormente o comprimento e freqüência das passadas, determinantes da velocidade do animal, assim como para descrições da postura do animal. A seguir, expomos alguns testes de desempenho desenvolvidos e já aplicados a animais arborícolas.

Locomoção sobre suportes ou superfícies horizontais: simulam o andar arborícola ("arboreal walking") sobre galhos (MARTIN, 1990; VIEIRA, 1995, VIEIRA, no prelo), variando apenas o diâmetro dos suportes e padronizando a rugosidade, textura da superfície e inclinação dos suportes. Quatro diâmetros diferentes são utilizados, variando de 1 a 4 polegadas. Os suportes são fixados na horizontal e o animal é estimulado a correr de uma extremidade a outra (VIEIRA, 1995; VIEIRA, no prelo; Figura 2).

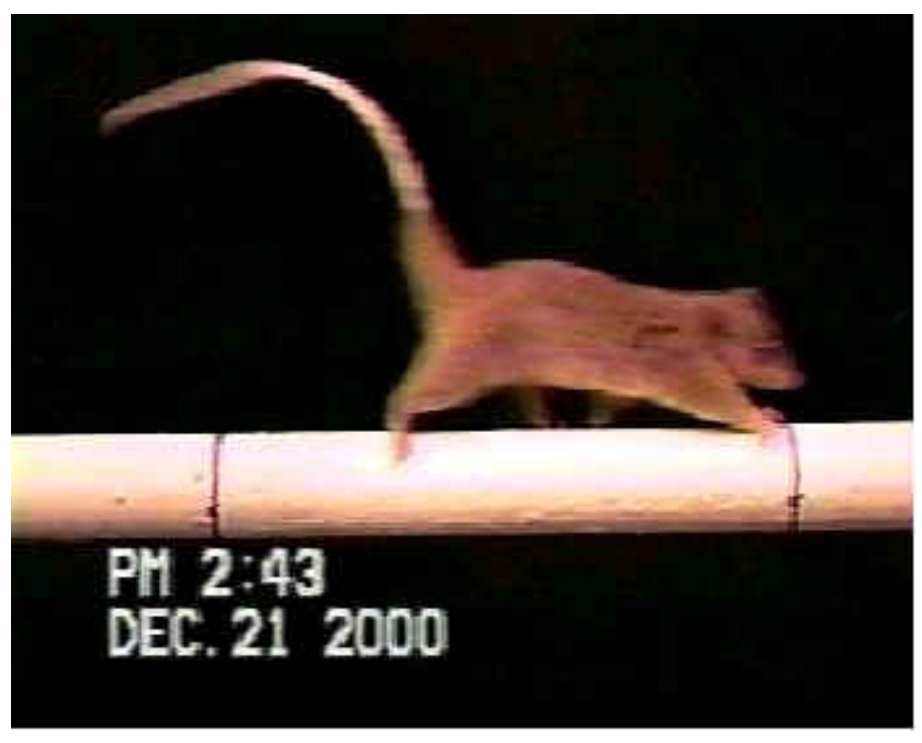

Figura 2: O marsupial Caluromys philander no teste de desempenho relativo ao suporte horizontal.

Locomoção sobre suportes verticais: Freqüentemente animais arborícolas utilizam pequenas árvores ou lianas para ter acesso ao topo de grandes árvores (CANT, 1992). O uso de suportes verticais como troncos, cipós e lianas, pode ser simulado com testes de desempenho utilizando-se cordas de três diâmetros diferentes. Elas ficam presas por uma roldana fixa no teto, e os animais são estimulados a subi-las (VIEIRA, 1995; VIEIRA, no prelo; Figura 3). 


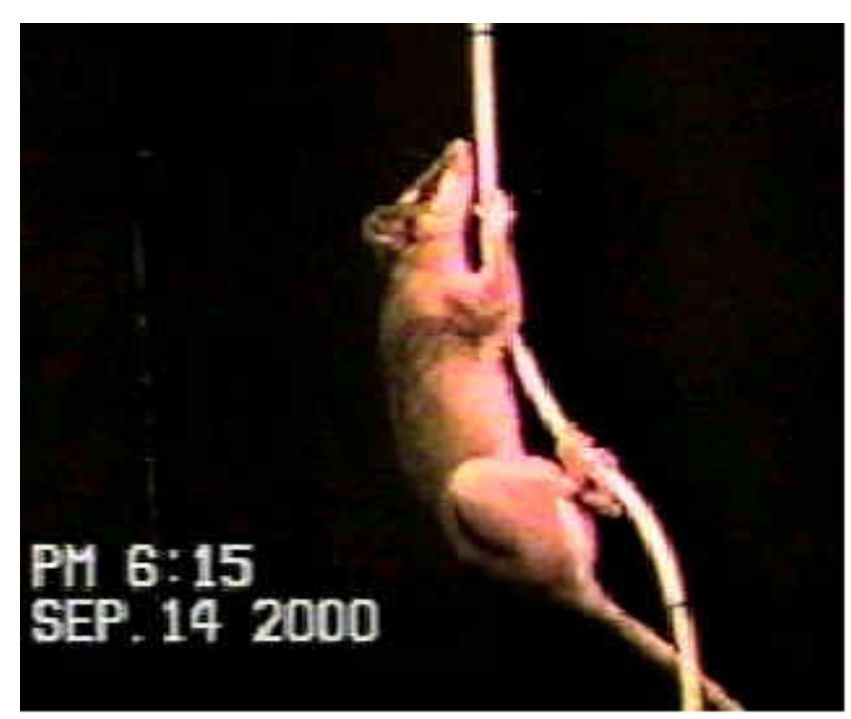

Figura 3: O marsupial Metachirus nudicaudatus no teste de desempenho relativo ao suporte vertical.

Habilidade de transpor descontinuidades entre suportes: São utilizados dois suportes de mesmo diâmetro ( 2 polegadas), um na horizontal e o outro em um ângulo de aproximadamente $60^{\circ}$. A distância entre eles é flexível, e o animal é estimulado a saltar distâncias cada vez maiores entre esses suportes, partindo de uma distância inicial de comprimento similar ao comprimento do corpo do animal (VIEIRA, 1995; VIEIRA, no prelo). As soluções possíveis encontradas pelos animais incluem numerosos padrões de salto.

\section{GERANDO E TESTANDO MODELOS ECOMORFOLÓGICOS}

A relação entre morfologia, desempenho (e.g., frequência e comprimento das passadas nos testes de locomoção arborícola) e ecologia envolve um grande número de variáveis. Um conjunto de hipóteses sobre relações entre variáveis caracteriza um modelo, que pode ser testado através de análise de caminhos ("path analysis") (MITCHELL, 1992). A análise de caminhos permite a divisão das correlações simples entre um grupo de variáveis de acordo com um modelo de trabalho sobre suas relações causais (por exemplo, $\mathrm{x}$ causa y; y por sua vez causa $\mathrm{z}$ ). $\mathrm{O}$ modelo pode ser expresso em um diagrama de caminhos ou transformado em uma série de equações lineares, que podem ser resolvidas através de Modelagem de Equações Estruturais, MEE ("structural equation modelling") (HOYLE, 1995). Além de proporcionar o teste de hipóteses e da sua capacidade de comparar modelos, essa técnica pode proporcionar o aperfeiçoamento de hipóteses, apontando para próximas observações ou experimentos críticos que podem aumentar a compreensão.

O primeiro passo seria estabelecer hipóteses a priori, antes da coleta dos 
dados, baseadas em princípios biomecânicos no caso da relação entre morfologia e desempenho locomotor, e no conhecimento prévio sobre a biologia da espécie ou das espécies. A seguir, estas hipóteses seriam formuladas em um diagrama de caminhos (como o da Figura 5, adiante).

Hipóteses alternativas resultariam em caminhos diferentes relacionando morfologia e desempenho locomotor. O ajuste dos dados a cada uma das hipóteses ( $a$ priori) alternativas pode ser testado usando MEE, e o modelo de melhor ajuste seria então favorecido.

O ajuste de diferentes modelos aos dados pode ser testado por um teste de chi-quadrado $\left(X^{2}\right)$. Entretanto, o objetivo não é descartar o modelo a ser testado, mas sim aceitá-lo. Assim, o modelo de melhor ajuste aos dados terá menor $X^{2}$ e uma maior probabilidade do desvio entre observado e esperado ser devido ao acaso. A diferença de $X^{2}$ entre modelos também segue uma distribuição de $X^{2}$, portanto podendo ser testada a significância de diferença de ajuste entre modelos. Os graus de liberdade são também a diferença entre os graus de liberdade dos dois modelos (HOYLE, 1995). Esta estatística é influenciada pelo número de parâmetros do modelo e, conseqüentemente, pelos graus de liberdade disponíveis. Uma forma de diminuir este efeito é comparar a razão $X^{2} / \mathrm{gl}$ entre os modelos.

A análise pode terminar neste ponto, ou simplificar o modelo a priori escolhido eliminando caminhos de efeito não-significativo. A partir deste ponto a análise passa a ser exploratória, e os novos modelos mais simples gerados são apenas novas hipóteses, que precisam de novos dados, independentes, para serem testadas.

Estes testes poderiam ser feitos em comparações entre espécies ou indivíduos. As comparações entre espécies necessariamente envolveriam uma maior escala de variação da morfologia e medidas do desempenho locomotor, o que seria vantajoso pela redução da magnitude relativa de erros de medida. A desvantagem seria a dependência dos dados, já que espécies diferentes são relacionadas entre si em diferentes graus, através de suas relações filogenéticas. Duas espécies podem ter morfologia e desempenho locomotor semelhantes, mas apenas devido à existência de um ancestral comum recente. Como separar então os aspectos da morfologia que são semelhantes devido à herança daqueles relacionados ao ambiente, isto é, à habilidade locomotora? Este problema, comum a qualquer tentativa de comparação estatística entre espécies, poderia ser abordado com o emprego de métodos comparativos filogenéticos, como constrastes independentes (DINIZ FILHO, 2000; FELSENTEIN, 1985; HARVEY \& PAGEL, 1991). Outra possibilidade seria testar as mesmas hipóteses através de comparações entre indivíduos e não espécies. A desvantagem na comparação entre indivíduos é que os erros de medida seriam maiores em relação à escala da variação, tanto da morfologia como das medidas de desempenho. 


\section{Uma aplicação: o caso do marsupial Philander frenata}

A metodologia descrita acima foi aplicada por VIEIRA (1995), em indivíduos adultos da cuíca cinza da Mata Atlântica, Philander frenata (Olfers, 1818) (PATTON, 1997) (Figura 4). Esses indivíduos foram capturados e mantidos em cativeiro para aclimatação, e submetidos à testes de desempenho locomotor sobre superfícies horizontais, já descritos anteriormente.

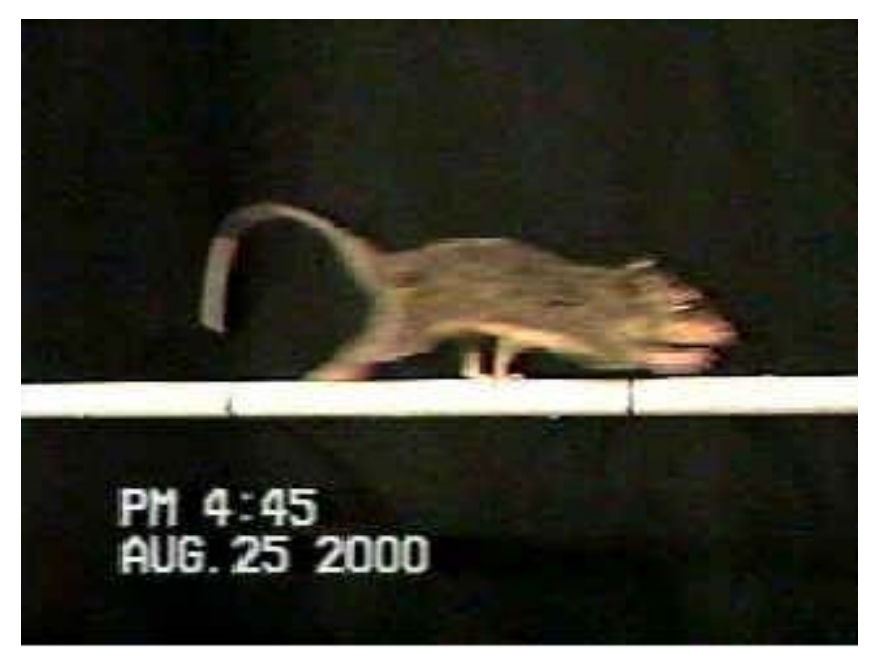

Figura 4: A espécie Philander frenata em um teste de desempenho.

As imagens dos animais foram analisadas, medindo-se o comprimento e freqüência da melhor seqüência de cada indivíduo, aquela em o animal se locomovia rápida e equilibradamente ao longo de todo o suporte. Portanto, quatro medidas de comprimento e freqüência de passada foram obtidas para cada indivíduo, uma para cada diâmetro de suporte. Para reduzir este número, foi realizada uma análise de componentes principais, ACP (MORRISON, 1976) na matriz de correlação das variáveis de desempenho. Os coeficientes de comprimento e freqüência de passada dos quatro suportes no primeiro componente principal (CP1) foram todos de mesma magnitude e sinal, indicando que CP1 representa um fator comum a todas as variáveis e que representa cerca de $78 \%$ da variação original. Foi interpretado como um fator determinante do comprimento e freqüência característico de cada indivíduo, independente do diâmetro do suporte utilizado. Os escores de cada indivíduo em CP1 de freqüência e comprimento da passada foram então utilizados na análise de caminhos.

As hipóteses formuladas a serem testadas na análise de caminhos eram: a velocidade máxima pode aumentar (1) pelo aumento no comprimento da passada e redução na freqüência, ou (2) pelo aumento da freqüência e redução do comprimento. A segunda hipótese poderia ser mais provável se o aumento do comprimento das passadas levar a um maior risco de escorregar e cair do galho (VIEIRA, 1995; VIEIRA, no prelo). 
Para testar essas hipóteses através da análise de caminhos, foram utilizados dois modelos simples, formulados a priori, das relações entre morfologia e desempenho no andar arborícola, um com caminhos para comprimento e freqüência de passadas (Figura 5), e outro com caminhos apenas para o comprimento da passada.

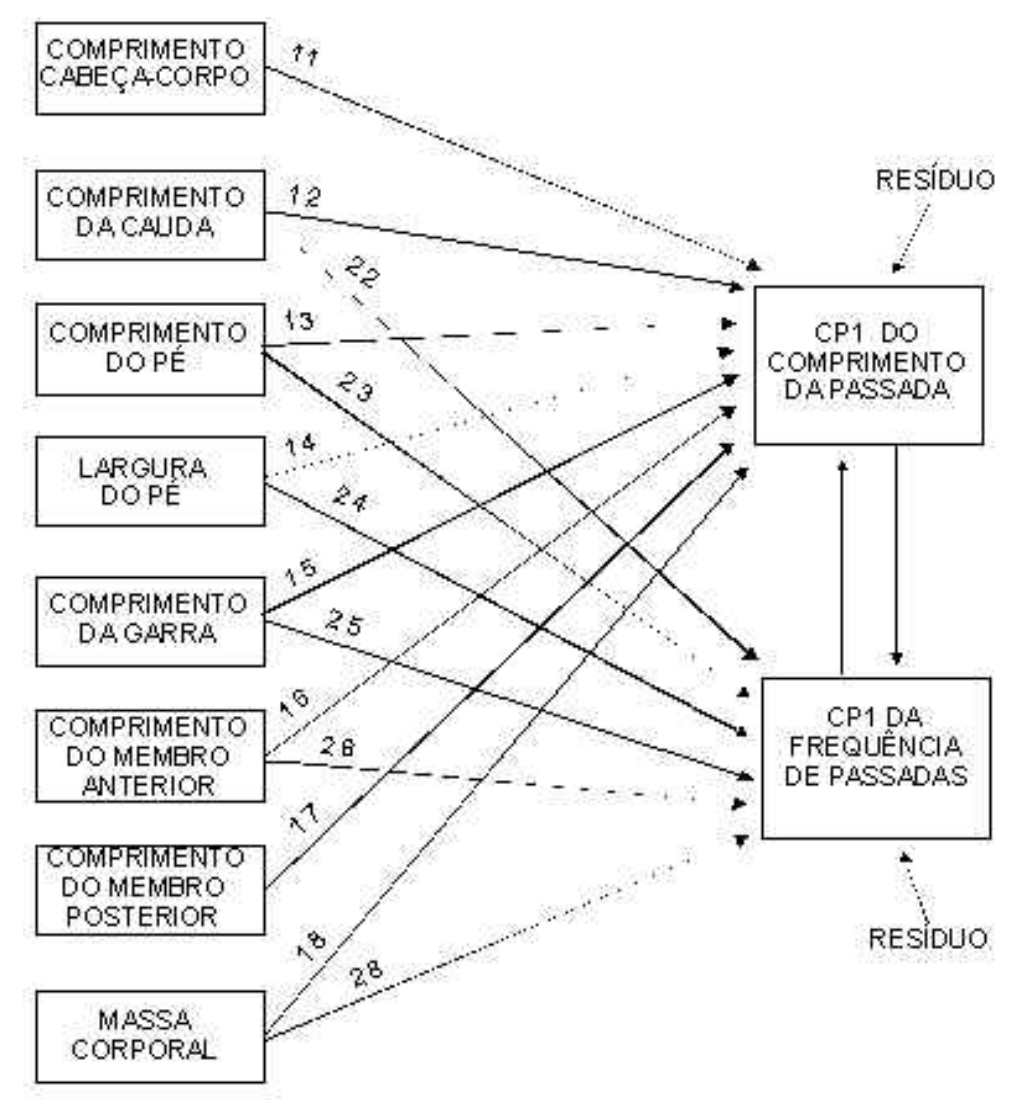

Figura 5: Modelo de relações entre variáveis da morfologia e de desempenho locomotor. Cada caminho ligando morfologia a desempenho tem um número próprio, correspondendo a um coeficiente de regressão partial $(\beta)$.

O resultado da análise de caminhos apoiou o modelo mais completo, com caminhos da morfologia afetando tanto o comprimento quanto a freqüência de passadas (Tabela 2).

\begin{tabular}{|c|c|c|c|c|c|c|}
\hline \multirow[b]{2}{*}{ Modelos } & \multicolumn{4}{|c|}{ Ajuste do modelo } & \multicolumn{2}{|c|}{$\begin{array}{l}\text { Diferença } \quad \text { de } \\
\text { ajuste em relação } \\
\text { ao } \quad \text { modelo } \\
\text { escolhido }\end{array}$} \\
\hline & $X^{2}$ & $\mathrm{~g} \mid$ & $P$ & $X^{2} / \mathrm{gl}$ & $\Delta X^{2}$ & $\Delta$ \\
\hline
\end{tabular}




\begin{tabular}{|c|c|c|c|c|c|c|c|c|}
\hline & & & & & & & $\mathrm{gl}$ & $P_{\text {dif }}$ \\
\hline \multicolumn{2}{|c|}{ A priori } & \multirow[b]{2}{*}{5.99} & \multirow[b]{2}{*}{3} & \multirow[b]{2}{*}{0.112} & \multirow[b]{2}{*}{2.00} & \multirow[b]{2}{*}{-} & \multirow[b]{2}{*}{ - } & \multirow[b]{2}{*}{ - } \\
\hline 1 & $\begin{array}{l}\text { *Caminhos } \\
\text { para } \\
\text { comprimento } \\
\text { e freqüência } \\
\text { de passadas }\end{array}$ & & & & & & & \\
\hline 2 & $\begin{array}{l}\text { Caminhos } \\
\text { somente para } \\
\text { comprimento } \\
\text { da passada }\end{array}$ & 21.67 & 9 & 0.010 & 2.41 & 18.09 & 6 & 0.005 \\
\hline 3 & $\begin{array}{l}\text { Modelo nulo: } \\
\text { nenhum } \\
\text { caminho } \\
\text { ligando } \\
\text { morfologia e } \\
\text { desempenho }\end{array}$ & 46.15 & 17 & 0.000 & 2.71 & 24.48 & 8 & 0.005 \\
\hline \multicolumn{2}{|c|}{ A posteriori* } & & & & & & & \\
\hline 4 & $\begin{array}{l}\text { Somente os } \\
\text { caminhos } \beta_{11} \\
\beta_{13} \beta_{14} \beta_{15} \\
\beta_{16} \beta_{17} \beta_{22} \\
\beta_{25}\end{array}$ & 13.60 & 9 & 0.137 & 1.51 & 7.61 & 6 & 0.280 \\
\hline & $\begin{array}{l}\text { mente o mo } \\
\text { ados no mod } \\
\text { oeficientes b }\end{array}$ & $a 1$ & 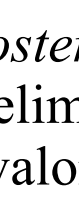 & ação & 200 & não- & & $\begin{array}{l}\text { trado, } \\
\text { tivos. }\end{array}$ \\
\hline
\end{tabular}

Eliminando os caminhos não significativos do modelo e considerando apenas aqueles com maior coeficiente, isto é, com maior efeito, chega-se a um modelo a posteriori (Figura 6). Este modelo sugere que o comprimento relativo das garras e da cauda são as variáveis que mais determinam a freqüência de passadas, enquanto o 
comprimento é mais afetado pelo comprimento relativo dos membros. Assim, indivíduos com garras relativamente longas, cauda curta, e membros posteriores longos teriam um melhor desempenho neste aspecto do nicho potencial (andar arborícola). Outros aspectos do nicho potencial devem ser medidos da mesma maneira. A posição relativa de cada individuo ou espécie no conjunto de medidas do nicho potencial seria uma medida da proximidade dos nichos.

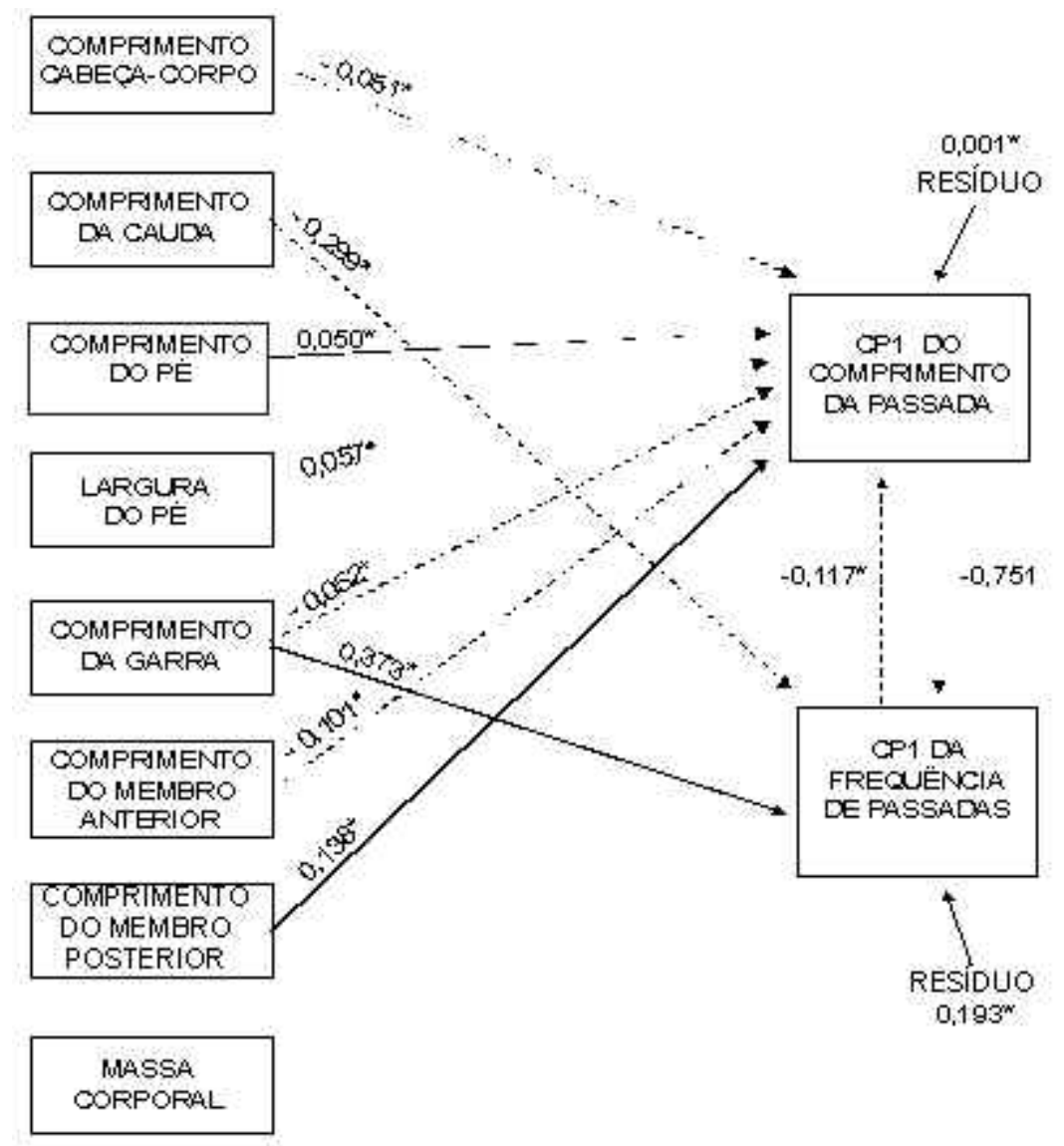

Figura 6: Modelo de relações entre variáveis da morfologia e de desempenho locomotor a posteriori.

Neste ponto, é preciso enumerar algumas considerações antes de partir para maiores conclusões:

(1) Um novo conjunto de dados seria necessário para testar a adequação do modelo escolhido, particularmente se for um modelo a posteriori. Este novo conjunto de dados poderia ser novos indivíduos da mesma espécie, de outra espécie, ou várias espécies. A significância do ajuste do modelo aos dados seria testado da mesma maneira. A generalidade do modelo será proporcional à variedade de espécies testada.

(2) Este método não demonstra se duas espécies competem ou não, mas pode indicar quais as têm mais chance de competir.

(3) Coeficientes positivos ligando uma medida morfológica ao desempenho não implicam 
necessariamente que os indivíduos com a morfologia mais adequada tenham maior sucesso reprodutivo, isto é, a seleção natural pode não estar favorecendo este aspecto da morfologia e do desempenho. A seleção age sobre o organismo como um todo, e não apenas sobre uma garra ou parte do corpo.

Concluindo, os testes de desempenho e a metodologia proposta podem gerar previsões quantitativas sobre os efeitos de diferenças morfológicas no nicho potencial de espécies ou indivíduos de gênero ou idade diferentes. Podem também ser utilizados para selecionar medidas morfológicas diretamente relacionadas a aspectos do uso habitat, e principalmente, determinar hipóteses ou modelos mais apoidas por um conjunto de dados. Testes de desempenho relacionados a outras atividades, como uso de recursos alimentares, podem também ser elaborados e combinados com análise de caminhos.

\section{REFERENCIAS}

CANT, G.H. Positional behavior and body size of arboreal primates: a theoretical framework for field studies and an illustration of its application. Am. J. Phys. Anthrop., n. 88, p. 273-283, 1992.

DINIZ FILHO, J.A.F. Métodos filogenéticos comparativos. Holos Editora Ltda, Ribeirão Preto, SP, 2000.

FELSENTEIN, J. Phylogenies and the comparative method. Am. Nat., n. 125, p. 1-15, 1985.

GARLAND, T., Jr.; LOSOS, J.B. Ecological morphology of locomotor performance in squamate reptiles. In: Wainwright, P.C.; Reilly, S.M. (eds.). Ecological morphology: integrative organismal biology. University of Chicago Press, Chicago, 1994, p240-302.

HARVEY, P.H.; PAGEL, M.D. The comparative method in evolutionary biology. Oxford University Press, New York, 1991, 239p.

HERNANDEZ, L.P.; MOTTA, P.J. Trophic consequences of differential performance: ontogeny of oral jaw-crushing performance in the sheepshead, Archosargus probatocephalus (Teleostei, sparidae). J. Zool., n. 243, p. 737-756, 1997.

HOYLE, R.H. (ed.). Structural equation modeling: concepts, issues, and applications. Sage Press, Thousand Oaks, California, 1995, 336p.

HUTCHINSON, G.E. Concluding remarks. Cold Spring Harbour Symp. Quant. Biol., n. 
22, p. 415-427, 1957.

LOSOS, J.B. Ecomorphology, performance capability, and scaling of West Indian Anolis lizards: an evolutionary analysis. Ecol. Monogr., n. 60, p. 369-388, 1990.

MARTIN, R.D. Primate locomotor patterns. In: Martin, R.D.; Martin, A.E. (eds.). Primate origins and evolution: a phylogenetic reconstruction. Princeton University Press,Princeton, New Jersey, 1990, p. 477-540.

MITCHELL, R.J. Path analysis: pollination. In: Scheiner, S.M.; Gurevitch, J. (eds.). Design and analysis of ecological experiments. Chapman and Hall, New York, 1993, p. 211-229.

MOERMOND, T.C. A mechanistic approach to the structure of animal communities: Anolis lizards and birds. Amer. Zool., n. 26, p. 23-37, 1986.

MORRISON, D.F. Multivariate statistical methods, $2^{\text {nd }}$ ed. McGraw-Hill, New York, 1976. 415p.

PATTON, J.L.; SILVA, M.N.F. Definition of species of pouched four-eyed opossums (Didelphidae, Philander). J. Mammal., n. 78, p. 90-102, 1997.

POUNDS, J.A. Ecomorphology, locomotion, and microhabitat structure: patterns in a tropical mainland Anolis community. Ecol. Monogr., n. 58, p. 299-320, 1988.

POUNDS, J.A. Habitat structure and morphological patterns in arboreal vertebrates. In: Bell, S.S., McCoy, E.D.; Mushinsky, H.R. (eds.). Habitat structure: the physical arrengement of objects in space. Chapman \& Hall, New York, 1991, p. 109-119.

RICKLEFS, R.E.; MILES, D.B. Ecological and evolutionary inferences from morphology: an ecological perspective. In: Wainwright, P.C.; Reilly, S.M. (eds.). Ecological morphology: integrative organismal biology. Chicago University Press, Chicago, 1994, p. 13-41.

SCHOENER, T.W. Mechanistic approaches to community Ecology: a new reductionism? Amer. Zool., n. 26, p. 81-106, 1986.

SCHOENER, T.W. The ecological niche. In: Cherrett, J.M. (ed.). Ecological concepts: the contribution of ecology to an understanding of the natural world. Blackwell Scientific, Oxford, England, 1989, p. 79-114.

VIEIRA, M.V. Ecomorphology of two neotropical marsupials: body size, form, climbing and walking the tight rope. Ph.D. Dissertation, University of Kansas, 
Lawrence, Kansas, U.S.A. 1995.

VIEIRA, M.V. no prelo. Locomoção, morfologia e uso do habitat em marsupiais didelfídeos: em busca de um modelo ecomorfológico. In: Cáceres, N.; MonteiroFilho, E.L.A. (eds.). Marsupiais Brasileiros. Editora Holos, Ribeirão Preto, SP.

WAINWRIGHT, P.C.; REILLY, S.M. (eds.). Ecological morphology. Integrative organismal biology. Chicago University Press, Chicago, 1994, 367p. 